\title{
GRADUATE STUDENTS SUPERVISED
}

Fordham University

$\begin{array}{ll}\begin{array}{l}\text { Touran Ipaktchi } \\ \text { John Orlando }\end{array} & \text { (M.S., 1974) } \\ \text { MacFord Zamore } & \text { (M.S., 1978) } \\ \text { Cecilia G. Unson } & \text { (M.S., 1978) } \\ \text { Deukjoon Kim } & \text { (Ph.D., 1978) } \\ \text { Moohi Yoon Kim } & \text { (Ph.D., 1979) } \\ \text { Bassam Nader } & \text { (Ph.D., 1980) } \\ \end{array}$

The Pennsylvania State University

Richard A. Gobao

(M.S., 1981)

Robert E. Anderson

(M.S., 1988)

Andrew M. Klos

(M.S., 1997)

Kira McGinty

(M.S., 1998)

Robert J. Gregg

(M.S., 2001)

Ann M. Bullion

(M.S., 2001)

Brian C. Raudenbush

(M.S., 2003)

David M. Dastrup

(M.S., 2003)

Jacob H. Waldman

(M.S., 2004)

Amy H. Yap

(M.S., 2006)

Hans F. Schmitthenner

(Ph.D., 1980)

John H. Dodd

(Ph.D., 1981)

Thomas R. Bailey

(Ph.D., 1983)

Jeremy I. Levin

(Ph.D., 1983)

James A. Gainor

(Ph.D., 1983)

Andrew Kucerovy

(Ph.D., 1984)

John E. Starrett, Jr.

(Ph.D., 1984)

Martin L. Bremmer

(Ph.D., 1984)

David M. Tschaen

(Ph.D., 1984)

Ravi S. Garigipati

(Ph.D., 1984) 
Ronald R. Staib

(Ph.D., 1985)

Stacey W. Remiszewski

(Ph.D., 1986)

Vincent J. Kalish

(Ph.D., 1986)

Richard P. Joyce

(Ph.D., 1986)

Edward Turos

(Ph.D., 1987)

Chakrapani Subramanyam

(Ph.D., 1987)

Thomas K. Hayes

(Ph.D., 1988)

Michael J. Melnick

(Ph.D., 1988)

Thomas A. Lessen

(Ph.D., 1990)

Rosanna Villani

(Ph.D., 1990)

Gary M. Lee

(Ph.D., 1990)

Scott I. Bell

(Ph.D., 1990)

Joseph Sisko

(Ph.D., 1991)

Michael D. Alexander

(Ph.D., 1991)

G. Davis Harris, Jr.

(Ph.D., 1993)

Matthias C. McIntosh

(Ph.D., 1993)

Janet L. Ralbovsky

(Ph.D., 1993)

Paul M. Scola

(Ph.D., 1993)

James J. Folmer

(Ph.D., 1994)

James R. Henry

(Ph.D., 1994)

Robert M. Borzilleri

(Ph.D., 1994)

R. Jason Herr

(Ph.D., 1994)

Glen T. Anderson

(Ph.D., 1995)

Christine S. Nylund

(Ph.D., 1995)

Geoffrey R. Heintzelman

(Ph.D., 1996)

Jian Jin

(Ph.D., 1997)

Daniel T. Smith

(Ph.D., 1997)

Gyoonhee Han

(Ph.D., 1997)

Matthew G. LaPorte

(Ph.D., 2000)

$\mathrm{Pu}$ Sun

(Ph.D., 2001)

Jeffrey E. Kropf

(Ph.D., 2002)

Xichen (Simon) Lin

(Ph.D., 2002)

Wenchun Chao

(Ph.D., 2004)

Gerald D. Artman, III

(Ph.D., 2004) 
Sungwoo Hong

(Ph.D., 2004)

Cuixiang (Sally) Sun

(Ph.D., 2005)

Pooja Aggarwal

Jeannie H. Jeong

Jason E. Camp

Jae Hong Seo

Matthew L. Meketa

Ilia Korboukh

Peng Liu

Praveen Kumar

Max M. Majireck

Joshua R. Sacher

\section{POSTDOCTORALS}

Fordham University

Joseph Auerbach

(1971-76)

Pratap Singh

(1974-76)

Satoshi Hibino

(1975-77)

Kimio Sugiura

(1975-76)

Michael F. Lipton

(1976-78)

Robert P. Hatch

(1976-79)

Anwer Basha

Jayant Shringarpure

(1976-78)

Fatima Z. Basha

Walter E. Pye

Paul Noire

The Pennsylvania State University

Walter E. Pye

Nazir Khatri

Christopher Wright

Tai-teh $\mathrm{Wu}$ 
Steven Nahm

Judith A. Morton

Kent Neuenschwander

Hideaki Natsugari

Joongik Yang

Sanker Chattapadyay

Ravi S. Garigipati

Donald M. Demko

Robert Cordova

William Studabaker

Mary A. Kinsella

Michihiko Noguchi

Bryan H. Norman

Eiichi Sugino

John P. Cherkauskas

Carolina Burgos Garcia

Kiyohiro Samizu

Charles E. Chase

Norbert Matzanke

Jesus de los Santos

Wen-Kui (Ken) Fang

Yung-hyo Koh

Osamu Irie

Kim M. Werner

Didier Stien

Stephen P. Keen

Jinhai Yang

Josef R. Bencsik

Douglas C. Bland

Grier A. Wallace

Joanne L. Pinder

Jon-Paul K. Meigh

Michael P. VanBrunt

Ivona R. Czuba Meigh
(1980-82)

(1981-83)

(1981-83)

(1983-84)

(1983-85)

(1984-88)

(1984-86)

(1984-86)

(1986-89)

(1986-89)

(1988-89)

(1989-91) (NIH Postdoctoral Fellow)

(1993-94)

(1993-95)

(1993-95)

(1995-97)

(1995-97) (NIH Postdoctoral Fellow)

(1996-97) (DFG Fellowship, Germany)

(1996-97)

(1997-98)

(1997-98)

(1997-98)

(1997-99)

(1998-99) (Lavoisier Fellowship, France)

(1998-00)

(1998-01)

(1999-01)

(1999-01)

(2000-02) (NIH Postdoctoral Fellow)

(2001-03)

(2001-03)

(2001-03)

(2001-02) 
Eunsun $\mathrm{Yu}$

(2002-04)

Magnus W.P. Bebbington

(2003-05)

Yogesh R. Mahajan

(2003-05)

Robert K. Orr

(2003-05)

Kristina Borstnik

(2005- )

Michael A. Evans

(2005- ) 